\title{
The Design of University Physical Education Management Framework Based on Edge Computing and Data Analysis
}

\author{
Wenze Sun and Yuchi Gao \\ Department of Physical Education, Teaching Center of Fundamental Courses, Ocean University of China, Qingdao 266000, China \\ Correspondence should be addressed to Yuchi Gao; sunwenze@ouc.edu.cn
}

Received 6 January 2021; Revised 9 February 2021; Accepted 23 February 2021; Published 13 March 2021

Academic Editor: Chi-Hua Chen

Copyright ( 2021 Wenze Sun and Yuchi Gao. This is an open access article distributed under the Creative Commons Attribution License, which permits unrestricted use, distribution, and reproduction in any medium, provided the original work is properly cited.

\begin{abstract}
Educational informatization has become the only way for mankind to enter the information age, and the application of educational resource library is a key issue in the integration of information technology disciplines. Aiming at the shortcomings of existing college physical education management technology, this article is dedicated to developing a new online management platform for physical education. Firstly, the introduction of edge computing technology in the architecture design of college physical education system can fully improve the efficiency of the management system. Secondly, based on data cleaning, the BP neural network algorithm modified by particle swarm is used to conduct in-depth analysis of the data of the university sports teaching management system and obtain corresponding optimization measures. Finally, it is verified in actual tests that the system can quickly perform data search, entry, modification, deletion, and other operations, which can ensure the realization of the school sports department's networked, scientific, standardized, and digitalized management of physical education resources.
\end{abstract}

\section{Introduction}

Information technology is changing the production, life, learning, and education methods of human society $[1,2]$. It not only brought a brand-new information environment to the education field, but also caused a comprehensive innovation of educational concepts, and had a profound impact on educational thinking, teaching methods and teaching methods [3]. The physical fitness of current students is declining year by year, which is closely related to the public physical education in colleges and universities $[4,5]$. In order to improve the level and effectiveness of university teaching, the research of public sports is very important, and we will carry out related research.

At present, many industries have moved towards office standardization, automation, and networking development, of course, including the physical education management department [6]. While encouraging teachers and students of various colleges and universities to engage in physical fitness activities, the education department also found that colleges and universities have corresponding drawbacks in sports management, such as the shortage of sports venues and unreasonable resource allocation [7]. In recent years, with the rapid development of computers, more and more companies, universities, and related departments use network and information management models [8]. Modern management has long become the trend of efficient operation in various industries. While reducing the pressure of managers, this model also provides convenience for people's lives [9]. While the construction of sports Informa ionization is carried out, information resources are also valued by sports organizations, and the development of an informationbased sports teaching management system is imperative [10]. Only in this way, sports resources will be distributed, and the needs of organizations and individuals at all levels for sports information will also be met. To achieve informatization and standardization of physical education management in colleges and universities, it must develop a physical education management system. Through the development of the student physical education management system, the physical education management system of colleges and universities realizes management Informa ionization and standardization [11, 12]. By improving the efficiency of sports information management, this will not only greatly 
improve the efficiency of student sports management information in colleges and universities but also further improve the physical fitness of students $[13,14]$.

Educational informatization has become the only way for mankind to enter the information age, and the application of the educational resource library is a key issue in the integration of information technology disciplines. Aiming at the shortcomings of existing college physical education management technology, this article is committed to developing a new online management platform for physical education. Develop a new university sports teaching management system by combining edge computing and particle swarmmodified BP neural network algorithm technology. Through this sports management system, the sports resource management of the school sports department can be scientific, standardized, and digitized. The system can quickly perform operations such as data search, entry, modification, and deletion.

\section{Overview of Related Technologies}

2.1. Overview of College Physical Education Management. Physical education classroom management is an important part of physical education activities and an important factor affecting the quality of classroom teaching. Herbart once said that "if you do not grasp the constraints of management firmly and gently, teaching any homework is impossible." From this, we can know the importance of scientific management in teaching $[15,16]$. Unlike other subjects, public physical education is taught outdoors to impart sports knowledge and skills so that students can form a lifelong physical education class [17]. In order to better complete the physical education teaching goals and improve the quality of teaching, physical education teachers control the impact of various potential factors in the classroom to complete the teaching goals, which requires teachers to have outstanding management capabilities [18]. Schematic diagram of the concept of college physical education management is shown in Figure 1.

In the process of public sports classroom teaching management, it is often impossible for both teachers and students to consider multiple tasks at the same time $[19,20]$. We must realize the psychological compatibility between teachers and students in the teaching process $[21,22]$. In the classroom teaching process, how teachers manage their work tasks and their attitudes towards students as well as how students manage their own learning tasks and their attitudes towards teachers' work are very important. In the teaching process, we learn from each other, combine the advantages of all aspects, and slowly optimize classroom teaching management. But while changing the concept of management methods, certain principles should be implemented [23]. In order to achieve informatization and standardization of physical education management in colleges and universities, it must develop a physical education management system. Through the development of the student physical education management system, the physical education management system of colleges and universities realizes management Informa ionization and standardization [24].
2.2. Edge Computing Technology. With the unprecedented popularity of mobile devices such as smart phones, cloudbased mobile sensors, tablets, and wearable devices, the Internet of Things has been extensively developed, thereby promoting the development of smart environments [25]. The Internet of Things enables mobile users to experience smart applications and enjoy various services with highquality service quality, such as mobile online games and augmented reality. These services usually require many mobile devices with limited size and low power consumption to perform computationally intensive and timesensitive tasks [26]. However, due to the low computing power and limited battery life of mobile devices, it is challenging to perform these services [27, 28]. Edge computing is an advanced version of cloud computing that reduces latency by bringing the service closer to the end user. Edge computing reduces the load of the cloud by providing resources and services in the edge network. However, edge computing will increase cloud computing by reducing the sensitivity of sensitive applications to end users. Like the cloud, edge service providers can provide end users with applications, data computing, and storage services [29].

Mobile edge computing (MEC) and wireless power transmission (WPT) are two promising technologies to solve the above challenges. In recent years, because MEC can significantly improve the computing power of mobile devices in a cost-saving and energy-saving manner, it has attracted increasing attention from the industry and academia. It allows mobile devices to offload some or all their computationally intensive tasks to MEC servers located at the edge of the wireless network, such as cellular base stations and access points. Unlike traditional cloud computing, MEC servers are deployed near end users [30]. Therefore, MEC has the potential to provide low-latency services, save energy for mobile users, and achieve high security. So far, many leading companies have regarded MEC as a promising technology for future wireless communication networks [31].

\section{Analysis of the Needs of College Physical Education Management}

We understand the background and significance of the physical education management system and the relevant research status at home and abroad. This chapter will analyze the needs of the physical education management system from the perspective of system function and nonfunctional needs analysis based on the content of the chapters. The overall framework of the system is planned, the system development platform is selected, and the relevant technology required for the development system is selected [32]. The college physical education management system uses information technology to efficiently manage the actual situation of physical education. It mainly includes competition management, physical fitness test management, physical education management, and user management. Through continuous investigation, in line with the principles of practicability, safety, and scientific, the demand analysis diagram of the college physical education management system is obtained, as shown in Figure 2 . 


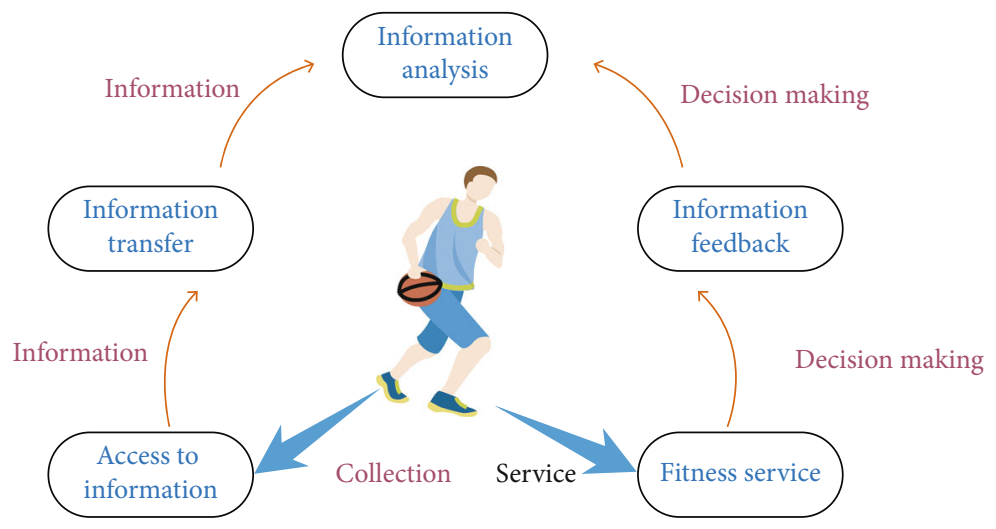

FIgURE 1: Schematic diagram of the concept of college physical education management.

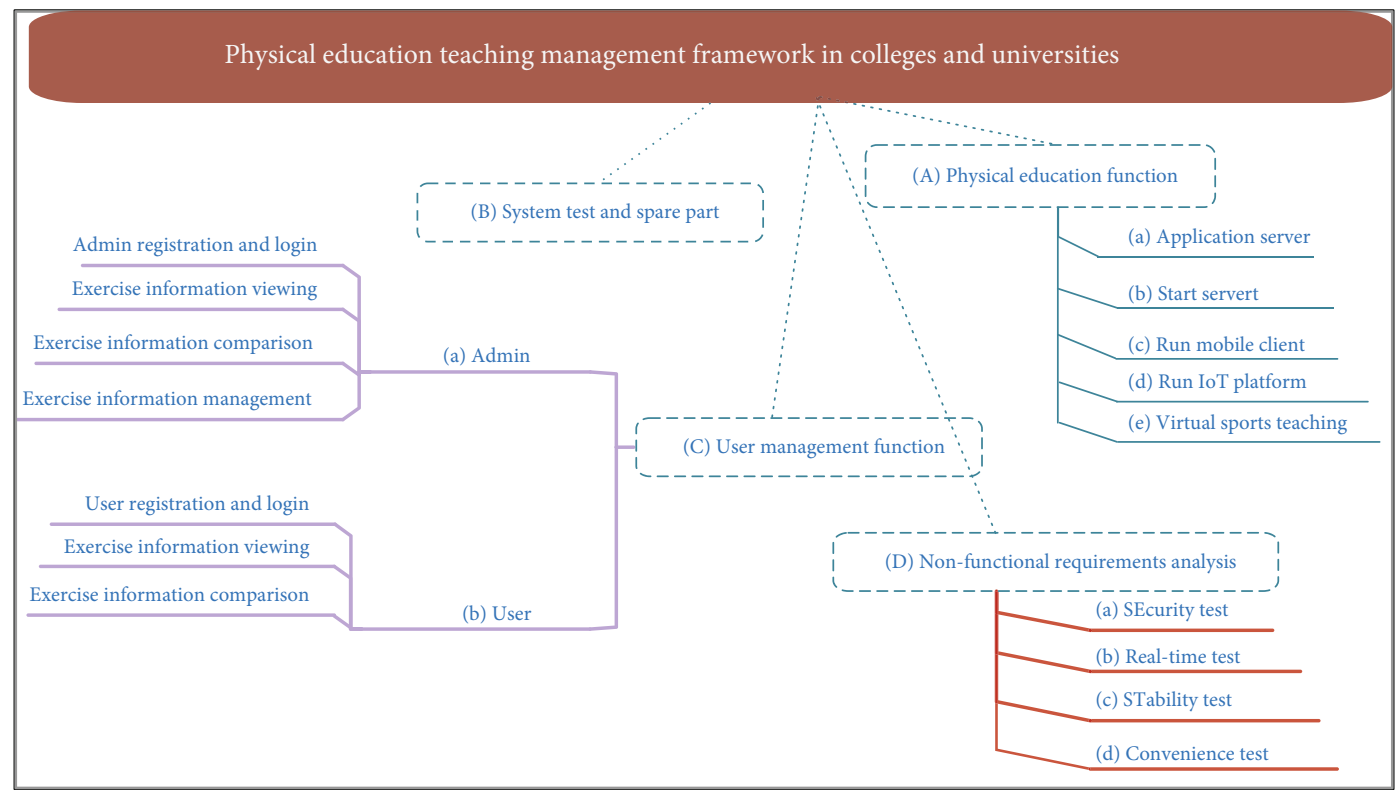

Figure 2: Distribution diagram of demand analysis of physical education teaching management in universities.

By strengthening and standardizing the work of physical education administrators, the fairness, orderliness, and efficiency of physical education management are guaranteed [33]. The demand analysis of the university sports management system in Figure 2 shows practical, safe, and scientific principles. From the current situation of the construction of public sports information and education resources in colleges and universities, the existing sports facilities and related sports electronic media resources have been greatly improved [34]. Some college sports departments or departments have certain experience in case handling, sports multimedia course teaching, and sports network distance teaching. The relevant sports webpages of the colleges and universities are also very refined and perfect. Some schools have also shared their own excellent sports courses with brother colleges and universities on the sports website page. However, due to the different levels of emphasis on different schools, there are still many colleges and universities in a backward situation in the update of sports information resources and video library webpages, and there are very few colleges and universities that realize the joint construction and sharing of public sports education resources between schools. Facing the needs of college physical education in public schools, there is an urgent need to develop a public sports resource sharing application platform system to enable the exchange of resources among colleges and universities, reduce redundant construction, and promote the informatization of college sports. The task system of the complete public sports resource sharing application platform differs from the nature of the unit. The system can be divided into several major functional modules through the summary and subtask system. Each part has specific functions and is divided into 3 major modules. The five relatively small parts of the part are teacher management module, student learning module, 
and administrator module. The small modules are sports online learning, student world, physical education teaching, teacher world, and administrator system.

\section{Analysis on the Management Data of Physical Education in Colleges and Universities}

Facing the needs of college physical education in public schools, there is an urgent need to develop a public sports resource sharing application platform system to enable the exchange of resources among colleges and universities, reduce redundant construction, and promote the informatization of college sports. In order to be able to compare with the original scheme, a simulation model based on simulation is introduced. The task system of the complete public sports resource sharing application platform varies with the nature of the unit. The hidden nodes of BP neural network are usually determined by repeated forward and backward propagation methods. By modifying or constructing training methods to change the number of hidden nodes, the corresponding initial weights and thresholds will also change, thereby affecting the network performance convergence and learning efficiency.

In order to reduce the impact, a neural network model based on particle swarm optimization is used to optimize the adjustment of weights and thresholds, thereby speeding up the convergence of the network and improving the learning efficiency of the network.

$$
\begin{aligned}
f 1(x) & =\frac{1}{1+e^{-\lambda x}} \\
f 1^{\prime}(x) & =\lambda f 1(x)[1-f 1(x)] .
\end{aligned}
$$

Suppose the neural network is trained to get a network with different weights and thresholds. After each training, the sample calculates the training error of the test sample and the test error of the test sample according to the formula.

$$
\begin{aligned}
E(w) & =\frac{1}{2} \sum_{k \in \text { outputs }}\left(t_{k}-o_{k}\right)^{2}, \\
F\left(X_{n+1} \mid J_{n}\right) & \neq F\left(X_{n+1} \mid J_{n}-Y_{n}\right) .
\end{aligned}
$$

After each training, BP algorithm and particle swarm optimization algorithm will jointly affect the adjustment of weights. Therefore, the basic BP network weight adjustment formula should also add a weight adjustment formula.

$$
\begin{aligned}
E\left(X_{n+1} \mid J_{n}\right) & \neq E\left(X_{n+1} \mid J_{n}-Y_{n}\right), \\
X_{t} & =\sum_{i=1}^{k} \alpha_{i} X_{t-i}+\sum_{i=1}^{k} \beta_{i} Y_{t-i}+u_{1 t} .
\end{aligned}
$$

By substituting the two coefficients into the equation of the second problem, we can get

$$
x^{(j+1)}=x^{(j)}+s_{j} d^{(j)},
$$

$$
\Delta f_{r}=\left[\frac{\partial f_{r}}{\partial x_{1}}, \frac{\partial f_{r}}{\partial x_{2}}, \cdots, \frac{\partial f_{r}}{\partial x_{n}}\right] .
$$

If the upper layer contains factor, the layer weight values are $b_{1}, b_{2}, b_{3} \cdots b_{n}$, respectively. In addition, we will calculate the composite weight of each layer element to the target layer.

$$
\begin{gathered}
\left|f^{(j)}-f^{(j-1)}\right| \leq \tau, \\
\left|f^{(j)}-f^{(b)}\right| \leq \tau, \\
F=\frac{\left(S S E_{r}-S S E_{u}\right) / k}{S S E_{u} /(T-2 k)} \sim F_{(k, T-2 k)}, \\
F\left(X_{n+1} \mid \Omega_{n}\right) \neq F\left(X_{n+1} \mid \Omega_{n}-Y_{n}\right) .
\end{gathered}
$$

Database design refers to the purpose of meeting data storage and processing requirements in a specific application environment and building a certain database model to effectively save data. In order to better complete the physical education teaching goals and improve the quality of teaching, physical education teachers control the impact of various potential factors in the classroom to complete the teaching goals, which requires teachers to have outstanding management capabilities. Database design is the core of the establishment of the system and also the basis for the establishment of the required database and application system. Combined with the actual situation of the physical education management of college, MySQL Server is used as a tool to build a database.

\section{College Physical Education Teaching Management Test and Evaluation}

Whether the university education and teaching management system can exert system efficiency, reduce production costs, and improve the system of management depend on the feedback after using the system. After design and development, the system enters the testing phase. Software testing is an important activity that is aimed at finding defects and reducing risks to ensure software quality, only through examples to test the system, according to the results of the operation, to evaluate the performance of the system. Types of physical education teaching management tests in colleges and universities-standard 1 are shown in Figure 3.

In the early and midstage of the construction of the college sports teaching management cluster, that is, when the data in the cluster has not reached the scale of fully training the diagnostic model, the fault diagnosis model can be provided by the real-time data management system in the cluster to provide some real-time collected bearing fault data set. Types of physical education teaching management test in colleges and universities-standard 2 are shown in Figure 4.

But it is still sufficient to complete the full training of the model. Currently, it is necessary to use migration learning to make edge computing end servers work together. Transfer learning between cloud and edge computing can greatly improve the accuracy of diagnosis. Through 


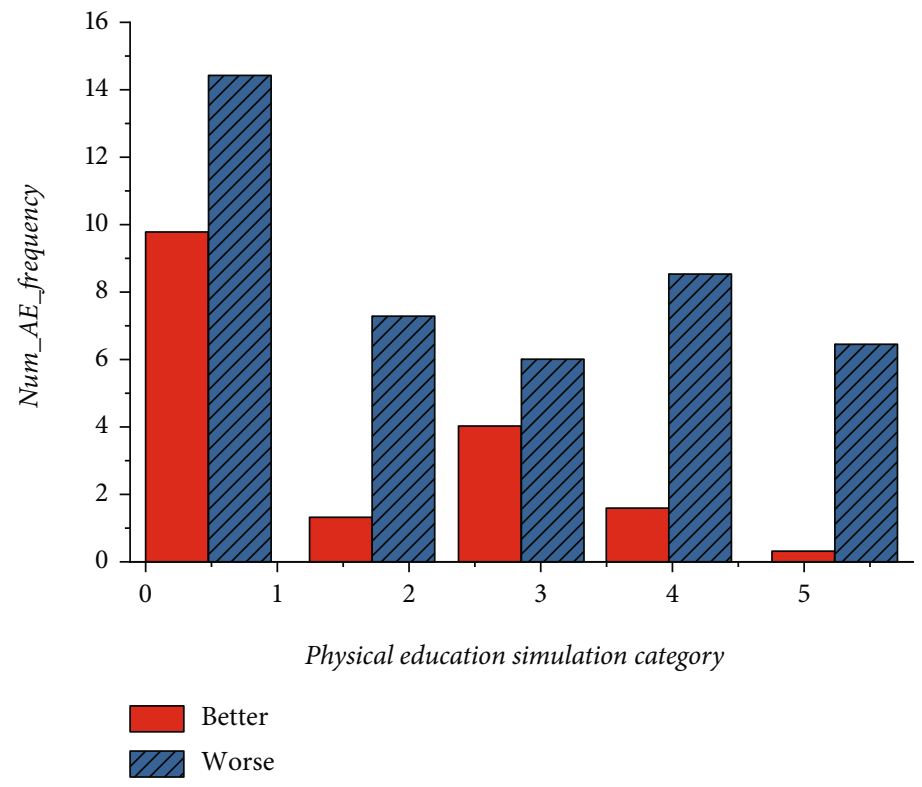

FIGURE 3: Types of physical education teaching management tests in colleges and universities-standard 1.

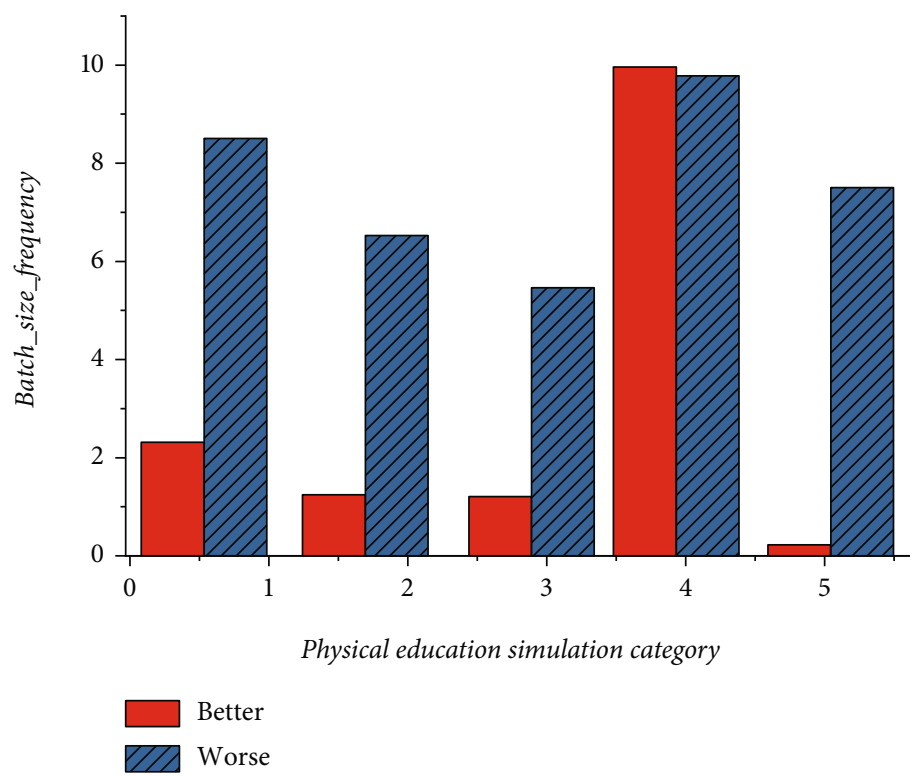

Figure 4: Types of physical education teaching management test in colleges and universities-standard 2.

exploration and experimentation, we can draw conclusions and use a small amount of training data in the initial stage of cluster construction. Changes with the number of MEC frequencies-energy consumption are shown in Figure 5.

At the edge end, only the local model needs to be updated with the model parameters trained on the fog end to accurately complete the real-time diagnosis of the bearing status. Changes with the number of MEC frequencies-number of people served are shown in Figure 6.

Carry out necessary tests for the design and implementation of the public sports education resource sharing platform system, and test the corresponding components. The build test is divided into two aspects: software and hardware to test. Build an independent test environment. Changes with the number of MEC frequencies-the number of packets transmitted are shown in Figure 7.

The running environment of the test needs to meet the public conditions. Next, conduct corresponding preinstallation tests on the public sports education resource sharing platform system. Perform individual tests on individual main test items. Changes with the number of MEC frequenciesexcellent evaluation are shown in Figure 8.

Through the above tests, it is found that most of the functions of the system can meet the system requirements. That 


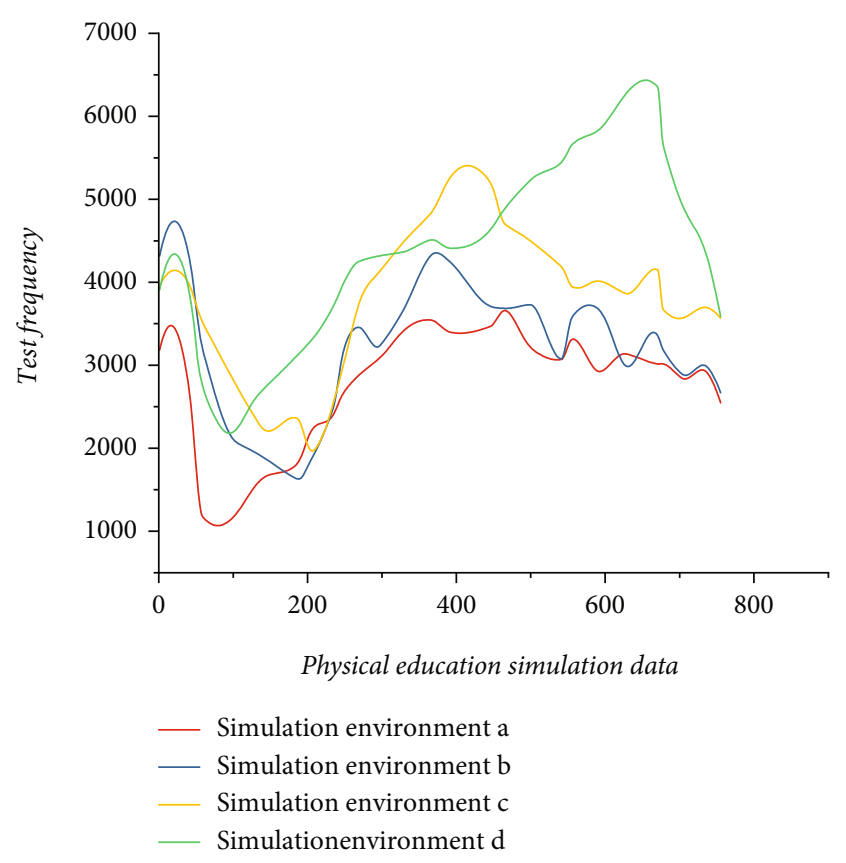

Figure 5: Changes with the number of MEC frequencies-energy consumption.

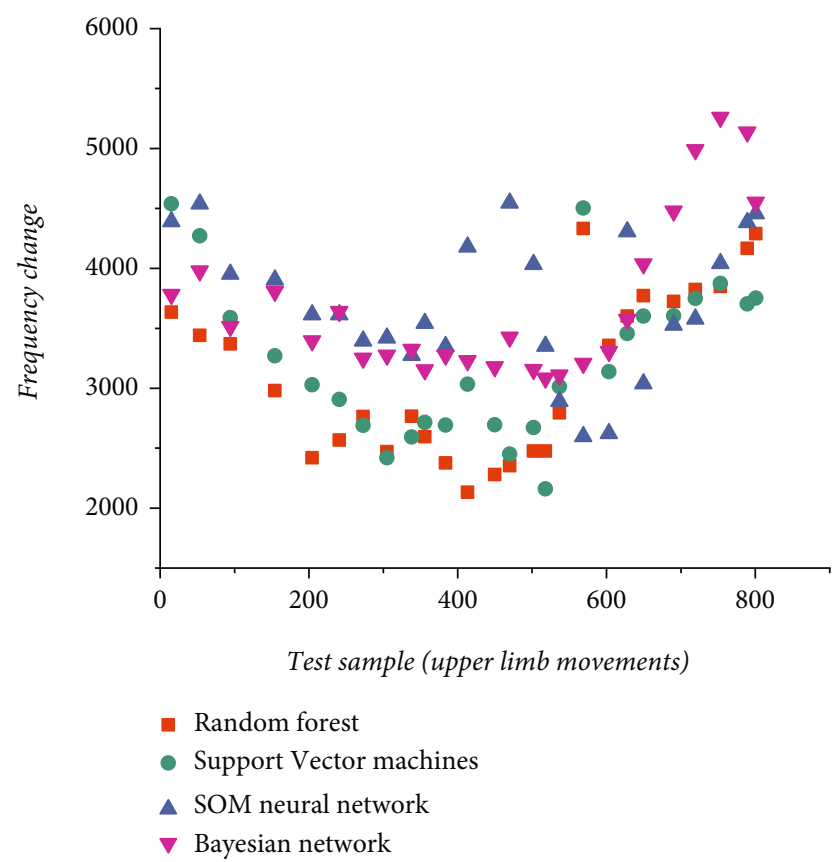

FIGURE 6: Changes with the number of MEC frequencies-number of people served.

is, the basic function of the system can satisfy the management of physical education. In terms of sports event registration, score entry, and related sports event publicity, the value of the sports education management system is gradually reflected, because many sports event publicity can be directly published to the system with the help of the system. Promoting the informatization of university sports plays an impor-

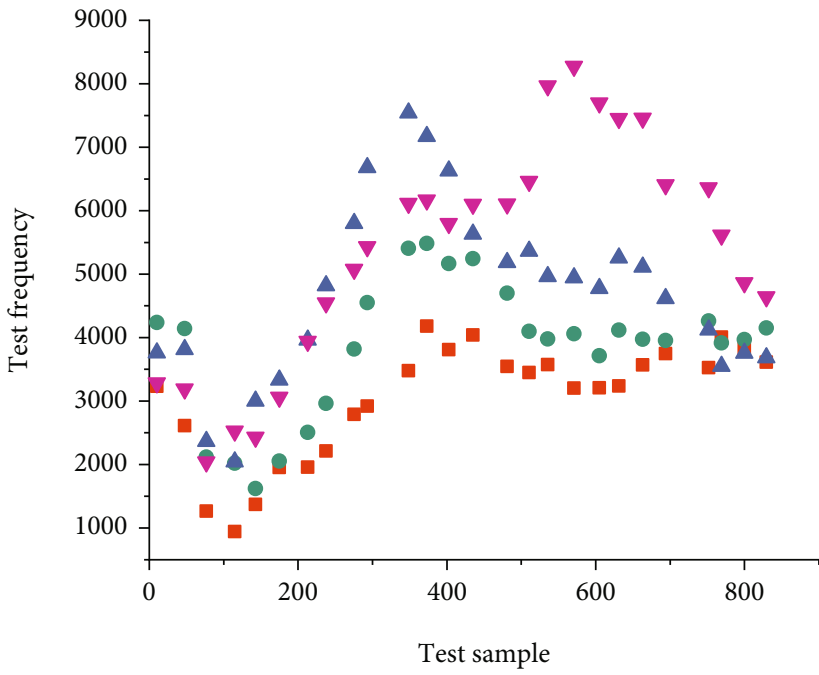

$\begin{array}{lll}\text { - Simulation category } 1 & \Delta \text { Simulation category } 3 \\ \text { - Simulation category } 2 & \nabla \text { Simulation category } 4\end{array}$

Figure 7: Changes with the number of MEC frequencies-the number of packets transmitted.

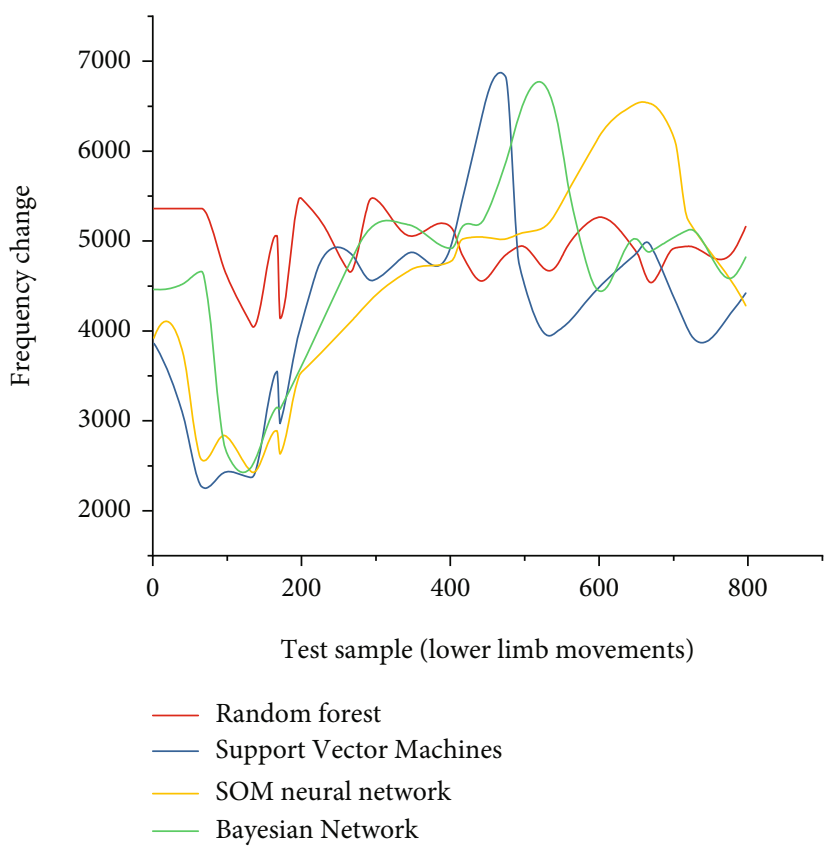

FIGURE 8: Changes with the number of MEC frequencies-excellent evaluation.

tant role. Facing the demand for physical education in public schools and universities, there is an urgent need to develop a public sports resource sharing application platform system to realize the exchange of resources between universities and reduce redundant construction. For example, the feedback information could not be fed back to the administrator in time, and there was a phenomenon that the registration could not be successful once during the registration 
process. But for the current test and use, no other errors have been found. We will also further analyze the related design process and debugging progress.

\section{Conclusion}

The college sports management system has realized management Informa ionization and standardization. This has not only greatly improved the efficiency of colleges and universities in student sports management information. This will also greatly improve the efficiency of sports information managers. Educational Informa ionization has become the only way for mankind to enter the information age, and the application of educational resource libraries is a key issue in the integration of information technology disciplines. Aiming at the deficiencies of existing university sports management techniques, this article is dedicated to developing a new sports online management platform. Combine edge computing and particle swarm-improved BP neural network algorithm technology to develop a new type of university sports teaching management system. The system can quickly perform operations such as data search, entry, modification, and deletion. The current sports management system still has certain shortcomings. For example, the feedback information cannot be fed back to the administrator in time, and there is a phenomenon that the registration is unsuccessful once during the registration process. But for the current test and use, no other errors were found. We will further analyze the related design process and debugging progress. In the future, we will further devote ourselves to sports management research, so as to ensure the realization of the network of these school sports departments, the scientific, standardized, and digitalized sports resource management.

\section{Data Availability}

We already included the analysis data in our manuscript.

\section{Conflicts of Interest}

The authors declare that there is no conflict of interests regarding the publication of this paper.

\section{References}

[1] X. Zhang, J. Zhang, Z. Liu, Q. Cui, X. Tao, and S. Wang, "MDP-based task offloading for vehicular edge computing under certain and uncertain transition probabilities," IEEE Transactions on Vehicular Technology, vol. 69, no. 3, pp. 3296-3309, 2020.

[2] W. Zhang, Z. Zhang, S. Zeadally, H. C. Chao, and V. C. M. Leung, "Energy-efficient workload allocation and computation resource configuration in distributed cloud/edge computing systems with stochastic workloads," IEEE Journal on Selected Areas in Communications, vol. 38, no. 6, pp. 1118-1132, 2020.

[3] K. Zhang, Y. Hu, F. Tian, and C. Li, "A coalition-structure's generation method for solving cooperative computing problems in edge computing environments," Information Sciences, vol. 22, no. 2, pp. 536-543, 2020.
[4] J. Zhang, L. Zhou, F. Zhou et al., "Computation-efficient offloading and trajectory scheduling for multi-UAV assisted mobile edge computing," IEEE Transactions on Vehicular Technology, vol. 69, no. 2, pp. 2114-2125, 2020.

[5] X. Xu, X. Liu, X. Yin, S. Wang, and L. Qi, "Privacy-aware offloading for training tasks of generative adversarial network in edge computing," Information Sciences, vol. 6, no. 11, pp. 345-356, 2020.

[6] X. Xiong, K. Zheng, L. Lei, and L. Hou, "Resource allocation based on deep reinforcement learning in IoT edge computing," IEEE Journal on Selected Areas in Communications, vol. 38, no. 6, pp. 1133-1146, 2020.

[7] X. Wang, Z. Ning, S. Guo, and L. Wang, "Imitation learning enabled task scheduling for online vehicular edge computing," IEEE Transactions on Mobile Computing, vol. 12, pp. 11-24, 2020.

[8] T. Wang, Y. Liang, Y. Yang et al., "An intelligent edgecomputing-based method to counter coupling problems in cyber-physical systems," IEEE Network, vol. 34, no. 3, pp. 16-22, 2020.

[9] M. C. I. Van Schalkwyk, M. Mckee, J. V. Been, C. Millett, and F. T. Filippidis, "Analysis of tobacco industry pricing strategies in 23 European Union countries using commercial pricing data," Tobacco Control, vol. 12, no. 4, pp. 126-132, 2019.

[10] Z. Sun and M. R. Nakhai, "An online learning algorithm for distributed task offloading in multi-access edge computing," IEEE Transactions on Signal Processing, vol. 99, p. 1, 2020.

[11] G. Sole, "University of Otago: towards specialisation with a Masters in Physiotherapy, endorsed in Sports Physiotherapy (continuing professional development series)," British Journal of Sports Medicine, vol. 51, no. 5, pp. 481-482, 2017.

[12] P. V. Rosen, I. M. Dohrn, and M. Hagströmer, “Association between physical activity and all-cause mortality: a 15-year follow-up using a compositional data analysis," Scandinavian Journal of Medicine \& Science in Sports, vol. 30, 2019.

[13] S. Rahimirad, M. Navaderi, S. Alaei, and M. H. Sanati, "Identification of hsa-miR-106a-5p as an impact agent on promotion of multiple sclerosis using multi-step data analysis," Neurological Sciences, vol. 3, no. 3, pp. 16-22, 2021.

[14] A. Mann, A. Mann, C. H. Tator, and J. D. Carson, "Investigation of knowledge and attitude about concussion diagnosis and management among family medicine residents," British Journal of Sports Medicine, vol. 51, no. 11, pp. A53.2-A5A53, 2017.

[15] J. Liu, H. Guo, J. Xiong, N. Kato, J. Zhang, and Y. Zhang, "Smart and resilient EV charging in SDN-enhanced vehicular edge computing networks," IEEE Journal on Selected Areas in Communications, vol. 38, no. 1, pp. 217-228, 2020.

[16] Z. Li, L. He, X. Tang, W. Han, and J. Wang, "Design and implementation of real assets analysis and evaluation system of power grid based on big data," Electrical Engineering, vol. 33, no. 4, pp. 134-147, 2019.

[17] S. Li, S. Lin, L. Cai, W. Li, and G. Zhu, "Joint resource allocation and computation offloading with time-varying fading channel in vehicular edge computing," IEEE Transactions on Vehicular Technology, vol. 69, no. 3, pp. 3384-3398, 2020.

[18] F. W. Leung, M. Koo, H. Jia et al., "Water exchange (WE) and quality improvement-enhanced advanced adenoma detection: a pooled data analysis of 6 randomized controlled trials," Journal of Clinical Gastroenterology, vol. 23, no. 3, pp. 34-47, 2020. 
[19] L. Yang, C. Zhong, Q. Yang, W. Zou, and A. Fathalla, “Task offloading for directed acyclic graph applications based on edge computing in Industrial Internet," Information Sciences, vol. 540, pp. 51-68, 2020.

[20] D. Kim, H. Lee, H. Song, N. Choi, and Y. Yi, "Economics of fog computing: interplay among infrastructure and service providers, users, and edge resource owners," IEEE Transactions on Mobile Computing, vol. 99, p. 1, 2019.

[21] M. Ke, Z. Gao, Y. Wu, X. Gao, and K. K. Wong, "Massive access in cell-free massive MIMO-based Internet of things: cloud computing and edge computing paradigms," IEEE Journal on Selected Areas in Communications, vol. 12, no. 3, pp. 321-330, 2021.

[22] D. L. Johnson, "Management of the anterior cruciate ligament deficient knee," Clinics in Sports Medicine, vol. 36, no. 1, pp. 654-662, 2017.

[23] J. Alent, D. M. Narducci, B. Moran, and E. Coris, "Sternal injuries in sport: a review of the literature," Sports Medicine, vol. 33, no. 4, pp. 134-147, 2019.

[24] J. M. Hung, X. Li, J. Wu, and M. F. Chang, "Challenges and trends in developing nonvolatile memory-enabled computing chips for intelligent edge devices," IEEE Transactions on Electron Devices, vol. 99, pp. 1-10, 2020.

[25] J. H. Huh, "Reefer container monitoring system using PLCbased communication technology for maritime edge computing," Journal of Supercomputing, vol. 33, no. 4, pp. 234-247, 2018.

[26] M. P. Hosseini, T. X. Tran, D. Pompili, K. Elisevich, and H. Soltanian-Zadeh, "Multimodal data analysis of epileptic EEG and rs-fMRI via deep learning and edge computing," Artificial Intelligence in Medicine, vol. 104, article 101813, 2020.

[27] M. A. Guillén, A. Llanes, B. Imbernón, R. Martínez-Espaa, and J. M. Cecilia, "Performance evaluation of edge-computing platforms for the prediction of low temperatures in agriculture using deep learning," Journal of Supercomputing, vol. 77, no. 9, pp. 145-153, 2021.

[28] M. Gairing and R. Savani, "Computing stable outcomes in symmetric additively separable hedonic games," Mathematics of Operations Research, vol. 12, no. 3, pp. 321-330, 2019.

[29] J. J. Fyfe and J. P. Loenneke, "Interpreting adaptation to concurrent compared with single-mode exercise training: some methodological considerations," Sports Medicine, vol. 48, no. 2, pp. 289-297, 2018.

[30] R. Du, Y. Liu, L. Liu, and W. Du, "A lightweight heterogeneous network clustering algorithm based on edge computing for 5G," Wireless Networks, vol. 26, no. 3, pp. 145-153, 2020.

[31] P. Derbeko, S. Dolev, and E. Gudes, "Wavelet-based dynamic and privacy-preserving similitude data models for edge computing," Wireless Networks, vol. 27, no. 1, pp. 351366, 2021.

[32] C.-H. Huang, L.-I. Hsu, T.-K. Chang et al., "Stress distribution of the patellofemoral joint in the anatomic $\mathrm{V}$-shape and curved dome-shape femoral component: a comparison of resurfaced and unresurfaced patellae," Knee Surgery Sports Traumatology Arthroscopy, vol. 25, no. 1, pp. 263-271, 2017.
[33] P. E. Boyle, M. D. Richardson, M. C. Savin, D. E. Karcher, and D. A. Potter, "Ecology and management of earthworm casting on sports turf," Pest Management Science, vol. 75, no. 8, pp. 145-153, 2019.

[34] H. Guo, X. Yang, and Y. Zhu, "Bloch theory-based gradient recovery method for computing topological edge modes in photonic graphene," Journal of Computational Physics, vol. 379, pp. 403-420, 2019. 